\title{
Mechanism for electron acceleration due to propagation of jets in the solar corona
}

\author{
R. Miteva, C. Vocks, G. Mann, \\ and H. Aurass
}

Astrophysicalisches Institut Potsdam, An der Sternwarte 16, 14482 Potsdam, Germany email: rmiteva@aip.de

\begin{abstract}
A kinetic analysis is performed here considering close relationship between plasma jets in the solar corona and a consequent acceleration of electrons. Solar jets are formed when a plasma stream is shooted out from a reconnection site. In some cases these jets are temporally and spatially associated with type III radio bursts, the latter being the radio signatures of nonthermal electron beam propagation through the corona. This theoretical work on jet associated type III burst argue the possibility of regarding the plasma jet as a trigger mechanism for electron energization (heating or/and acceleration). While the jet propagates in the solar corona, it creates wave-like disturbances in the background plasma, that are in general unstable. Further on we are interested in how a test electron will behave in such (electrostatic) wave field. The settings for the jet and the test electron are done under typical coronal conditions.
\end{abstract}

Keywords. Sun: corona, acceleration of particles, X-rays: solar jets

\section{Introduction}

In the solar corona, jets are streams of hot plasma expelled from a reconnection site. The reconnection model is one of the possible explanation for the generation of the jets, Yokoyama \& Shibata (1995). When magnetic field lines with opposite direction approach each other and reconnect, the re-arrangement of the new magnetic configuration shoot plasma away in the corona, forming the observed with Yohkoh/SXT solar jets, Shibata et al. (1994).

Additionally number of authors performed data analysis on whether solar jet can be closely associated with propagation of non-thermal electron beams in the solar corona (so-called type III/U burst in the radio spectrum), see Aurass et al. 1994, Pick et al. 1994, Kundu et al. 1995, Raulin et al. 1996.

In the present paper, we argue whether electron acceleration is possible due to the propagation of plasma jets in the solar corona. Our aim is to look for the possibility and the favourable conditions for a solar jet to be regarded as a trigger of electron heating or/and acceleration. We apply the kinetic approach to one special configuration of jet aligned to the magnetic field lines and additionally the excited wave-like disturbance propagates in the same direction. Thus we restrict the general problem up to an electrostatic and collisionless case.

\section{Kinetic approach}

\subsection{Dispersion relation for electrostatic ion-acoustic waves}

We start the present study from the linearized Vlasov-Maxwell equations for describing the evolution of the electric field vector $\mathbf{E}$. We assume that the jet is aligned to the magnetic field lines and so we approximate the general problem considering only a $1 \mathrm{D}$ 
case (say only $z$-component). Additionally, the excited wave-like disturbances in the plasma due to the jet propagation are assumed to travel only along the $z$-direction. Thus the general problem is reduced to electrostatic one that is given with the following algebraic equation:

$$
\varepsilon_{33} E_{z}=0
$$

where the component of the dielectric tensor, see Baumjohann \& Treumann (1997), reads

$$
\varepsilon_{33}=1-2 \pi \sum_{s} \frac{\omega_{\mathrm{p}, s}}{k^{2}} \int_{-\infty}^{\infty} \int_{0}^{\infty} v_{\perp} \frac{k \partial f_{s} / \partial v_{\|}}{k v_{\|}-\omega} d v_{\perp} d v_{\|} .
$$

In the last equation the plasma frequency is denoted with $\omega_{\mathrm{p}, s}=\left(4 \pi N_{0, s} e^{2} / m_{s}\right)^{1 / 2}$, with number density $N_{0, s}$, elementary charge $e$, mass $m_{s}$ - for each plasma species (subscript $s)$. As usual, $\omega$ and $k$ represent the frequency and the wave number, respectively. Here the velocity distribution function $f_{s}$ is depending on the particle velocities parallel $v_{\|}$ and perpendicular $v_{\perp}$ with respect to the ambient magnetic field. In order to take into account the initial jet velocity, we assume a drifting Maxwell distribution,

$$
f_{s}=\frac{1}{\left(2 \pi v_{\mathrm{th}, s}^{2}\right)^{3 / 2}} e^{-\left[\left(v_{\|}-v_{0, s}\right)^{2}+v_{\perp}^{2}\right] / 2 v_{\mathrm{th}, s}}
$$

with thermal velocity $v_{\mathrm{th}, s}=\left(k_{\mathrm{B}} T_{s} / m_{s}\right)^{1 / 2}$, where $k_{\mathrm{B}}$ is the Boltzmann constant and $T_{s}$ is the temperature of each plasma species. The initial jet velocity is denoted with $v_{0, s}$. After solving the integrals, we obtain the following expression for the general dispersion relation of electrostatic waves,

$$
k^{2}=-\sum_{s} \frac{\omega_{\mathrm{p}, s}^{2}}{v_{\mathrm{th}, s}^{2}}\left[1+\xi_{s} Z\left(\xi_{s}\right)\right] .
$$

The plasma dispersion function $Z\left(\xi_{s}\right)$ and $\xi_{s}$ are defined as:

$$
Z\left(\xi_{s}\right)=\frac{1}{\pi^{1 / 2}} \int_{-\infty}^{\infty} \frac{e^{-x^{2}}}{x-\xi_{s}}, \quad x=\frac{v_{\|}-v_{0, s}}{2^{1 / 2} v_{\mathrm{th}, s}}, \quad \xi_{s}=\frac{v_{\mathrm{ph}}-v_{0, s}}{2^{1 / 2} v_{\mathrm{th}, s}}
$$

with $v_{\mathrm{ph}}=\omega / k$ is the phase speed.

\subsection{Jet-plasma interaction}

The interaction of the jet with the background plasma (solar corona) is considered as a plasma with 4 components (species), i.e. an electron-proton plasma for both the jet and background plasma. We introduce the following quantities for the jet:

$$
v_{\mathrm{e}}^{\mathrm{jet}}=v_{\mathrm{p}}^{\mathrm{jet}}=v_{0} \quad N_{\mathrm{e}}^{\mathrm{jet}}=N_{\mathrm{p}}^{\mathrm{jet}}=\nu N_{0} \quad T_{\mathrm{e}}^{\mathrm{jet}}=\theta T_{0} \quad T_{\mathrm{p}}^{\mathrm{jet}}=T_{0},
$$

and for the background plasma

$$
v_{\mathrm{e}}^{\mathrm{b}}=v_{\mathrm{p}}^{\mathrm{b}}=0 \quad N_{\mathrm{e}}^{\mathrm{b}}=N_{\mathrm{p}}^{\mathrm{b}}=(1-\nu) N_{0} \quad T_{\mathrm{e}}^{\mathrm{b}}=T_{\mathrm{p}}^{\mathrm{b}}=T_{0},
$$

where $N_{0}=1.12 \times 10^{9} \mathrm{~cm}^{-3}$ is the total number density (for typical frequency of around $300 \mathrm{MHz}), T_{0}=1.4 \mathrm{MK}$ is the coronal temperature, and the parameters of the problem are: $0<\nu<1, \theta=2-7$ and $v_{0}=(0.002-0.22) v_{\text {th }, \mathrm{e}}, v_{\text {th }, \mathrm{e}}=4600 \mathrm{~km} \mathrm{~s}^{-1}$ (consistent with the reported physical parameters of the jets by Shimojo \& Shibata (2000)). After normalizing the plasma parameters to $N_{0}, T_{0}, v_{\mathrm{th}, \mathrm{e}}, \omega_{\mathrm{p}, \mathrm{e}}$ and $\lambda_{\mathrm{D}, \mathrm{e}}=v_{\mathrm{th}, \mathrm{e}} / \omega_{\mathrm{p}, \mathrm{e}}$, the Debye length, we obtain the dimensionless four-component dispersion relation, describing the propagation of ion-acoustic electrostatic waves in this plasma configuration:

$$
k^{2}=-\left[(1-\nu) H\left(\xi_{\mathrm{e}}^{\mathrm{b}}\right)+(1-\nu) H\left(\xi_{\mathrm{p}}^{\mathrm{b}}\right)+\frac{\nu}{\theta} H\left(\xi_{\mathrm{e}}^{\mathrm{jet}}\right)+\nu H\left(\xi_{\mathrm{p}}^{\mathrm{jet}}\right)\right],
$$


where $H(\xi)$ is defined by

$$
H(\xi)=1+\xi Z(\xi)
$$

with the corresponding sub- and superscripts.

\section{Numerical results}

We solve the four-component dispersion relation and we present the result for one special choice of the parameters, namely: $\theta=5.5\left(T_{\mathrm{e}}^{\mathrm{jet}}=7.7 \mathrm{MK}\right), \nu=0.9\left(N_{\mathrm{e}, \mathrm{p}}^{\mathrm{jet}}=\right.$ $1.08 \times 10^{-9} \mathrm{~cm}^{-3}$ ) and $v_{0} \equiv v_{\text {jet }}$ (covers an interval of values). From figure 1 is seen that instability (positive imaginary part of the wave frequency, $\gamma=\operatorname{Im}(\omega)>0$ ) is present only for small range of jet velocities, i.e. $0.09 v_{\text {th,e }}<v_{0}<0.15 v_{\text {th,e }}$ (or $415<v_{0}<690 \mathrm{~km} \mathrm{~s}^{-1}$ according to our chosen parameters). Shimojo \& Shibata (2000) reported on jet with observed parameters covering the calculated ones.

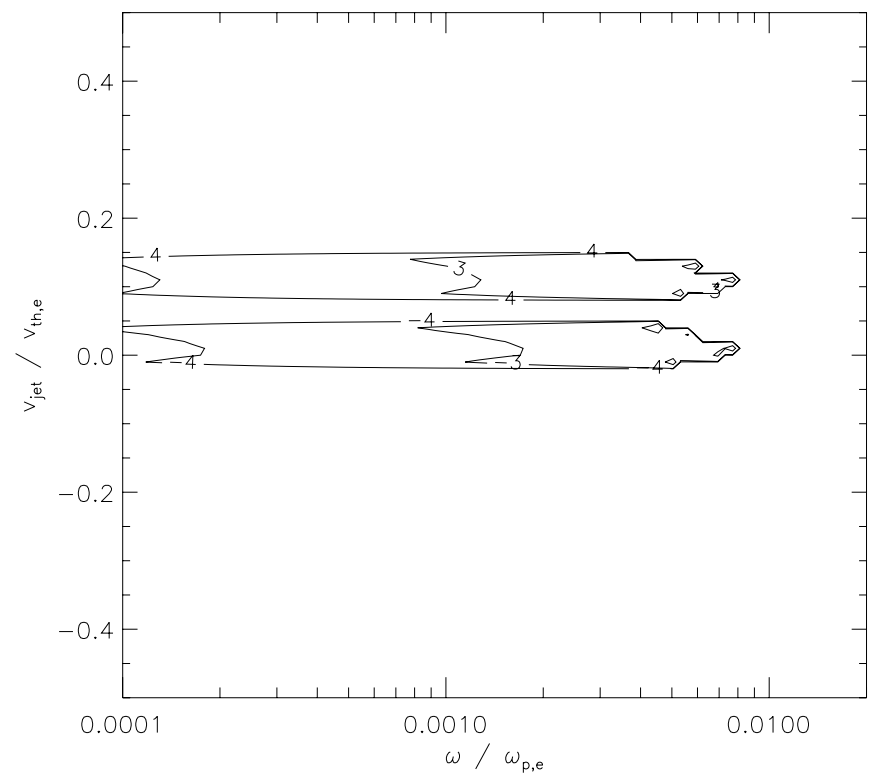

Figure 1. Jet velocity-real frequency plot, the imaginary part of the frequency is given with isolines: damping is represented with negative numbers on the plot, while the growth rate is given with positive numbers, i.e. $\gamma=10^{-(4)}-10^{-(3)}$.

\section{Test electron movement}

The numerical calculations from the previous section give that the excited electrostatic fluctuations/waves in the jet-plasma configuration are subject to instability and growth rate of the order of $10^{-3}$ is expected. In order to study the influence of the electrostatic wave on the electron, we proceed further following the behaviour of a test electron in such a wave field. We introduce the relativistic equations of motion:

$$
\dot{p}=-e \frac{\partial \phi}{\partial z} \quad v=\dot{z},
$$

with $p=m_{0} v / \sqrt{1-(v / c)^{2}}$, $v$, particle velocity, $c$, velocity of light and for the spatialtemporal behaviour of the electrostatic wave field we choose $\phi(t)=e^{\gamma t} \cos [k z-\omega t]$. For 

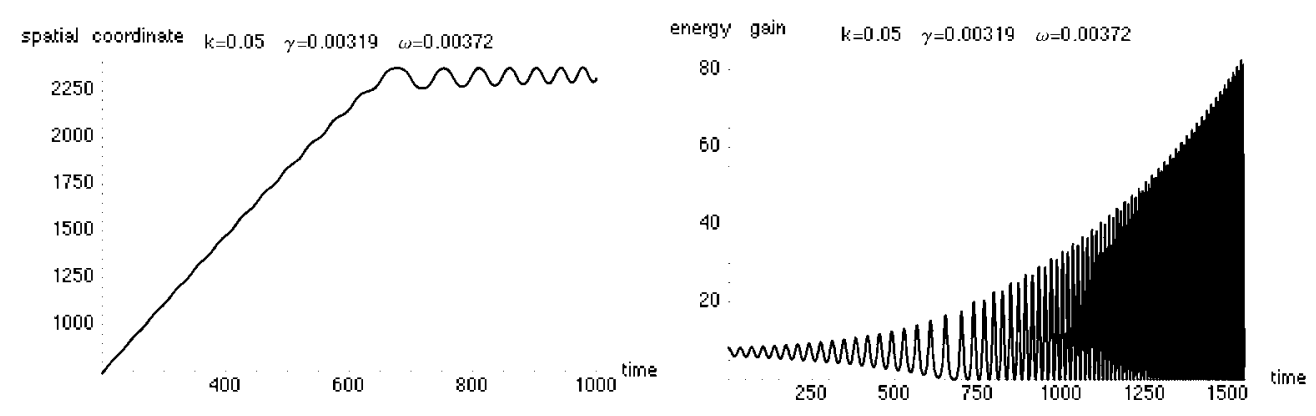

Figure 2. (left) Spatial-temporal movement of the test electron (trapping into the electrostatic wave); (right) Energy-time diagram (represents energy gain of the test electron).

the relativistic kinetic energy we have

$$
W=m_{0} c^{2}\left[\frac{1}{\sqrt{1-(v / c)^{2}}}-1\right] .
$$

From figure 2 we can quantitatively notice that the test particle is trapped into the wave field (left) and gains energy of the order of $80 k_{\mathrm{B}} T \approx 10 \mathrm{keV}$ for $0.8 \mu \mathrm{s}$ (right).

\section{Discussions}

From the performed kinetic analysis on jet-plasma interaction we can conclude that electrostatic waves (from ion-acoustic type) can be excited and for certain values of the plasma parameters, intability could also occur. As seen from figure 1 the intability is present only for a narrow range of all possible jet velocities. During the time while the instability is acting, suprathermal electron (with velocity greater than $4 v_{\text {th,e }}$ in order to be consistent with the assumed collisionless regime) can be trapped into the wave field. While propagating further with the wave, the electron can gain energy of the order of typical type III electron in the solar corona $(\approx 8 \mathrm{keV})$. Because the jet velocity decrease with time (the kinetic energy of the jet is transferred toward the electron), the instability cannot be sustained and eventually will switch-off. Thus the energized electron could freely leave the intability region. Finally such electron could give rise to type III/U bursts in the radio spectrum.

\section{Acknowledgements}

R. M. is supported by DAAD under grant number Ref. 324 A/03/10336.

\section{References}

Yokoyama, T. \& Shibata, K. 1995, Nture 375, 42

Shibata, K., Yokoyama, Y. \& Shimojo, M. 1994, in: S. Enome \& T. Hirayama (eds.) Proc. Kofu Symposium (NTRO Rep. No. 360) 75

Aurass, H., Klein, K.-L. \& Martens, P.C.H. 1994, Solar Phys., 155, 203

Pick, M., Raoult, A., Trottet, G., Vilmer, N., Strong, K. \& Magalhaes, A. 1994, in: S. Enome \& T. Hirayama (eds.) Proc. Kofu Symposium (NTRO Rep. No. 360) 263

Kundu, M.R. Raulin, J.P., Nitta, N., Hudson, H.S., Shimojo, M, Shibata, K. \& Raoult, A. 1995, ApJ (Letters) 447, L135

Raulin, J.P., Kundu, M.R., Hudson, H.S., Nitta, N. \& Raoult, A. 1996, A\&A 306, 299

Baumjohann, W. \& Treumann, R.A. 1997, Basic space plasma physics, Imperial College Press

Shimojo, M. \& Shibata, K. 2000, ApJ 542, 1100 